\title{
Software-defined elastic optical networks
}

\author{
Jason Jue • Vincenzo Eramo • Víctor López • \\ Zuqing Zhu
}

Published online: 18 June 2014

(C) Springer Science+Business Media New York 2014

Recent advances in flexible and programmable device technologies, such as flexible-spectrum variable-rate transceivers and reconfigurable optical add-drop multiplexers (ROADMs), coupled with emergence of software-defined network paradigms, such as OpenFlow, have enabled the possibility of software-defined elastic optical networks that can be flexibly and dynamically provisioned and reconfigured. The ability to flexibly manage and provision resources allows service providers to support a wide range of emerging dynamic highbandwidth applications, including data center networking, cloud computing, and optical network virtualization.

In developing mechanisms for supporting the rapid and flexible provisioning of resources in elastic optical networks, several key challenges must be considered. These challenges may include issues related to the cost-effective design of data and control plane architectures, the intelligent provisioning and reconfiguration of resources to support dynamic and heterogeneous applications, the design of mechanisms to provide survivability against equipment failures and major disasters, and the design of architectures and protocols for reducing energy consumption in elastic optical networks.

The purpose of this Special Issue is to have solicited submissions of original work and survey papers on all topics

\section{J. Jue}

Department of Computer Science, The University of Texas at Dallas, Richardson, TX 75080, USA

V. Eramo $(\varangle)$

DIET, Sapienza University of Roma, 00184 Rome, Italy

e-mail: Vincenzo.Eramo@uniroma1.it

\section{López}

Telefónica I+D, S.A.U., 28006 Madrid, Spain

Z. Zhu

School of Information Science and Technology, University of Science and Technology of China (USTC), Hefei, Anhui, China related to recent advances in elastic optical network and software-defined optical networks. The Special Issue consists of five papers. The first paper is a survey article on Software Defined Optical Network. The next paper focuses on the proposal and evaluation of a new Elastic Optical Network (EON) paradigm based on Spatial, Spectral and Temporal domain. The third paper proposes and evaluates new mapping techniques of Virtual Networks in EON. The last two papers focus on the experimental evaluation of restoration mechanisms in EONs. Brief summaries of the accepted articles are listed below.

The paper titled "Software-Defined Optical Networks (SDONs) - A Survey" by P. Bhaumik et al. gives an overview of SDONs. It then explains the benefits and challenges of extending SDNs to multi-layer optical networks, including flexible grid and elastic optical networks, and how it compares to Generalized Multi-Protocol Label Switching (GMPLS) for implementing a unified control plane. An overview on the industry and research efforts, SDON standardization and implementation is also given next. Finally, the paper outlines the benefits achieved by SDONs for network operators, and also some of the important and relevant research problems that need to be addressed.

The paper titled "Software Defined Elastic Optical Networking in Temporal, Spectral, Spatial Domains" by S. J. B. Yoo et al. proposes and investigates the performance of the 3-Dimensional (3D) Elastic Optical Networking paradigm in which the information is carried in the temporal, spectral and spatial domains. The authors use dynamic Optical Arbitrary Waveform Generation (OAWG) and Optical Arbitrary Waveform Measurement (OAWM) for transmitters and receivers respectively to exploit elasticity in the temporal and spectral domains. The spatial domain is supported by carrying on a fiber more light beams with different orbital angular momentums (OAM). The introduced OAM multiplexing 
and demultiplexing are demonstrated with the realization of a Photonic Integrated Circuit (PIC). The authors also describe how a 3D-EON- based network can be controlled by an Open Flow-based Control Plane and present results from an experimental test-bed in which the proposed solutions have been implemented.

The paper titled "Upgrade-aware Virtual Infrastructure Mapping in Software-Defined Elastic Optical Networks" by $Z$. Ye et al. investigates a new problem of efficient Virtual Infrastructure (VI) mapping in Elastic Optical Network. The authors address the issue related to the Upgrade Virtual Topology (UVT) mapping when users want to expand their business by requesting more nodes/links and high computing/bandwidth resources. They study how the support of this added service impacts the Physical Substrate (PS) providers' revenue by introducing two different pricing policies. It also discusses how the integration of Software Defined Elastic Optical Network can effectively support VI mappings with upgrade as it provides flexibility and programmability in transmission and switching elements while abstract control plane intelligence from the hardware to a centralized node.

The paper titled "Multipath Restoration and Bitrate Squeezing in SDN-based Elastic Optical Networks" by F. Paolucci et al. proposes the use of bitrate squeezing and multipath restoration in the context of dynamic SDN controlled EONs. The authors formally state the bitrate squeezing and multipath restoration problem and then model it by introducing an Integer Linear Programming (ILP) formulation. The objective is to maximize the amount of restored bitrate by exploiting the available spectrum resources also along multiple routes. As a result of the stringent time to computing a solution, a heuristic algorithm providing better trade-off between optimality and complexity is proposed to solve the problem. The proposed solution is implemented in an SDN control plane and OpenFlow extensions are presented and implemented to control the Sliceable Bandwidth Variable Transponders and Bandwidth Variable Optical Cross Connects in the EONs.

The paper titled "Multi-Stratum Resources Resilience in Software Defined Data Center Interconnection based on IP over Elastic Optical Networks" by H. Yang et al. proposes a novel multi-stratum resource resilience (MSRR) architecture for SDN-EON-based data center interconnection networks. The MSRR architecture can enable joint optimization of IP network, elastic optical network and application stratum resources, and enhances the service resilience and the data center responsiveness to the dynamic end-to-end service demands. Additionally, a service-aware resource collaborative resilience (RCR) strategy for MSRR is introduced based on the proposed architecture. This can provide restoration using the multiple stratums resources in case of optical node failures. The overall feasibility and efficiency of the proposed architecture are evaluated by simulation and experimentally verified on an experimental testbed with the OpenFlow-enabled devices.

The Guest Editors thank all the authors who submitted papers to the Special Issue and also acknowledge all the reviewers for ensuring a high quality of the selected papers.

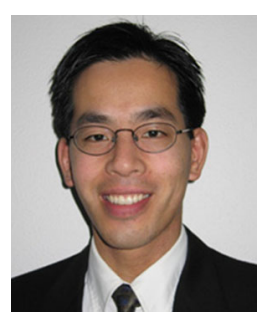

Jason Jue received the B.S. degree in Electrical Engineering and Computer Science from the University of California, Berkeley in 1990, the M.S. degree in Electrical Engineering from the University of California, Los Angeles in 1991, and the Ph.D. degree in Computer Engineering from the University of California, Davis in 1999. He is currently a Professor in the Department of Computer Science at the University of Texas at Dallas. His current research interests are in the area of optical networks and network survivability. He has served as an Associate Editor of IEEE/OSA Journal of Optical Communications and Networking and currently serves on the Editorial Boards of IEEE Communications Surveys and Tutorials and Springer Photonic Network Communications. Dr. Jue was a recipient of the NSF CAREER Award in 2002, and is the co-author of papers that received the Best Paper Award at IEEE Globecom 2005, ONDM 2010, and IEEE ICC 2011.

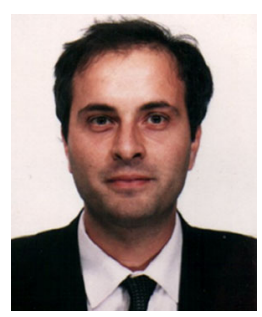

Vincenzo Eramo received his "Laurea" degree in Electronics Engineering in 1995 and his "Dottorato di Ricerca" (PhD degree) in Information and Communications Engineering in 2001, both from the University of Roma "La Sapienza". Currently he is an Aggregate Professor in Department of Engineering of Information, Electronics and Telecommunications. His research activities have been carried out in the framework of national and international projects. In particular Vincenzo Eramo was scientific coordinator for University of Roma "La Sapienza" in EURO (Experimental University Router Open), BORA-BORA (Building Open Router Architecture Based on Router Aggregation) and SFINGI (SoFtware-routers to Improve Next-Generation Internet), three national projects financed by MUR (Minister for University and Research) in 2002-2004, 2005-2006 and 2011-2013 respectively and focused on the performance evaluation of Software Router. Vincenzo Eramo was scientific coordinator for University of Roma "La Sapienza" of E-PhotoONe+ and BONE, two Networks of Excellence focusing on the study of Optical Networks and financed by European Commissions (FP6 and FP7) in 2006-2007 and 2008-2011 respectively. He has been an Associate Editor of IEEE Transactions on Computer since July 2011 and an Associate Editor of Photonic Network Communications (Springer) since September 2013. He is an Editorial Board member of Dataset Papers in Optics (Hindawi) since November 2011, The Scientific World JournalComputer Science (Hindawi) since October 2013 and Open Journal of Optics and Photonics (Herbert Open Access Journal) since July 2013. He was a Guest Editor of the following Special Issues: "Open Source for Networking: Protocol Stack" (March 2014) organized on IEEE Network, "Innovative Techniques for Power Consumption Saving in Telecommunication Networks" (February 2014) organized on Journal of Electrical and Computer Engineering. Actually he is a Guest Editor of the following Special Issues:, "Open Source for Networking: Tools and Applications" (September 2014) organized on IEEE Network e "Software Defined Elastic Optical Networks" (September 2014) organized on Photonic Network Communications. He was Chairman in some international conferences (IEEE ICC 2003, EuroIMSA 2005, IEEE ICC 2011). 


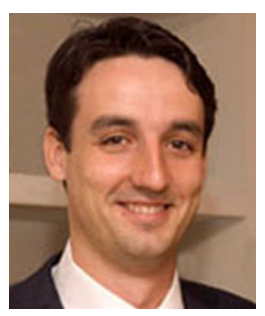

Víctor López received the M.Sc. degree in telecommunications engineering from Universidad de Alcalá de Henares, Spain, in 2005 and the Ph.D. degree in computer science and telecommunications engineering from Universidad Autónoma de Madrid (UAM), Madrid, Spain, in 2009. The results of his $\mathrm{Ph} . \mathrm{D}$. thesis were awarded with the national COIT prize 2009 of the Telefónica foundation in networks and telecommunications systems. In 2004, he joined Telefónica I+D as a Researcher, where he was involved in next generation networks for metro, core, and access. He was involved with several European Union projects (NOBEL, MUSE, MUPBED). In 2006, he joined the High-Performance Computing and Networking Research Group (UAM) as a Researcher in the ePhoton/One+ Network of Excellence. He worked as an Assistant Professor at UAM, where he was involved in optical metro-core projects (BONE, MAINS). In 2011, he joined Telefonica I+D as Technology specialist. He has co-authored more than 100 publications and contributed to IETF drafts. His research interests include the integration of Internet services over IP/MPLS and optical networks and control plane technologies (PCE, SDN, GMPLS).

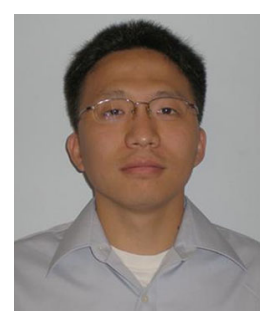

Zuqing Zhu received the $\mathrm{PhD}$ degree from the Department of Electrical and Computer Engineering, University of California, Davis, in 2007. From July 2007 to January 2011, he worked in the Service Provider Technology Group of Cisco Systems, San Jose, as a senior R\&D engineer. In January 2011, he joined the University of Science and Technology of China (USTC), where he currently is an Associate Professor. He has published more than 100 papers in peer-reviewed journals and conferences. He has been in the technical program committees (TPC) of INFOCOM, ICC, GLOBECOM, ICCCN and etc. He is also an editorial board member of Elsevier Journal of Optical Switching and Networking, Springer Telecommunication Systems Journal, Willey European Transactions on Emerging Telecommunications Technologies, and etc. He has received the Best Paper Awards from the IEEE International Conference on Communications (ICC) 2013, the IEEE Global Communications Conference (GLOBECOM) 2013, and the IEEE International Conference on Computing, Networking and Communications (ICNC) 2014. He is a Senior Member of IEEE and also a Senior Member of OSA. 